\title{
Postvaccinal neurological complications
}

\section{Complicações neurológicas pós-vacinais}

\author{
Getúlio Daré Rabello
}

\section{Case report}

CHCYL, female, white, 30 years old. On May $21^{\text {st }} 2010$ the patient was vaccinated against H1N1 flu. After few days, she developed neurological signs characterized by paresthesias in the left side of the body. Subsequently she developed central vestibular syndrome, with hiccups and uncontrollable vomiting. The patient was admitted to the Hospital Samaritano on June $8^{\text {th }} 2010$ and the MRI has showed lesions of demyelinating and inflammatory character in the medulla oblonga and high cervical spinal cord. Cerebrospinal fluid (CSF) examination has showed lymphocytic pleocytosis. In addition, she developed pancreatitis, confirmed with MRI of the abdomen. After receiving dexamethasone she improved. In early August 2010, her condition got worse, starting with gait ataxia, complete horizontal ophthalmoplegia, intense paresthesia and areflexia in the left upper limb. The patient was re-admitted and the MRI has showed worsening of inflammatory demyelinating lesions. CSF maintained the previous characteristics.

She had received methylprednisolone pulse $1 \mathrm{~g}$ / EV for 5 consecutive days and then she was discharged with oral prednisone, which was withdrawn in a few months, with reversal of the situation.

In November 2011, the neurological disorder was presented again and it was characterized by central vestibular syndrome and bilateral internuclear ophthalmoplegia. The new MRI showed new lesions with the same character in the pons. It was conducted research of antiacquaporin-4 antibody in the blood, which was negative.

Facing this evolution, diagnose was defined: Multiple Sclerosis. The question is: the vaccine is directly responsible for all problems ("prolonged" ADEM) or it simply deflagrated an immune disease that was underlying?

This doubt, that we had in this particularly case, is the keynote of the most neurological complications after vaccination. In this exhibition we will try to set the most current data about this issue.

\section{A bit of history}

Until the introduction of antibiotics, infectious diseases were responsible for the great scourges of humanity, not even respecting social classes. Many historical figures died because of infectious diseases. Even after the advent of antibiotics, diseases that do not respond to them, such as viruses, continued to leave its trace in humanity.

On May 14, 1796, Edward Jenner inoculated James Phipps, an eight years old boy, with pus taken from a pustule of Sarah Nemes who was a milkmaid and had suffered from cowpox (skin disease characterized by reddish vesicles; this virus is similar to smallpox disease but much more severe and often fatal; it occurs through contact with infected cow udders; when the patient recovers from cowpox, he is immune to smallpox). The boy contracted an infection extremely benign and ten days later he got recovered. Months later, Jenner inoculated Phipps with smallpox pus. The boy did not get sick. Thus the vaccine was introduced. The term vaccine is derived from the Latin word vacca.

\section{Immunization ${ }^{1,2}$}

There are two types of immunization: active and passive. Active immunization occurs when the individual's immune system gets in contact with a foreign substance to the body, it responds by producing antibodies and immune cells ( $\mathrm{T}$ lymphocytes). This type of immunity usually lasts for several years, sometimes for a lifetime. The two ways to acquire active immunity are by contracting an infectious disease or by vaccination.

Passive immunization is achieved in transferring the antibodies produced by an animal or other man. This type of immunity produces a quick and efficient protection. However, it is only temporary, lasting on average of few weeks or months. There isn't formation of immune memory or long-term immunity. The natural passive immunity is the most common type of passive immunity, characterized by the passage of antibodies from mother to fetus through the placenta. This transfer of antibodies occurs in the last two months of pregnancy, in order to give a good immunity to children during their first year of life. The artificial passive immunity can be acquired in three main forms: the combined human immunoglobulin, hyperimmune human immunoglobulin and heterologous serum. Blood transfusion is another way to get passive immunity, as virtually all types of blood products (e.g. whole blood, plasma, packed red cells, platelet concentrate, etc.) contain antibodies. 


\section{Vaccines ${ }^{1,2}$}

The vaccine is an immunobiological product containing one or more immunizing agents (isolated vaccine or in combination) under different ways: live attenuated bacteria or viruses, inactivated viruses, killed bacteria and components of infectious agents purified and/or chemically or genetically modified. The goal is to mimic the primary immune response leading to development of protective and long-term immunological memory.

\section{Classification of vaccines}

\section{Toxoid vaccines:}

As our main examples are vaccines for tetanus, diphtheria, botulism, cholera, besides of some infections such as pertussis. The vaccine is developed with components produced by agents. There are disadvantages for such vaccine: they usually require adjuvants and multiple doses. Besides of that, local reactions are common, due to adjuvants or reactions of type III (Arthus), determining erythema and induration at the injection site. This reaction is because of antibody excess at the site, aggregating to toxoids molecules and activating complement.

\section{Killed/inactivated vaccines}

These are compound of inactivated microorganisms, which means that they are no longer alive so they were unable to multiply. Many aspects make them as similar as toxoid type vaccines. The immune response to an inactivated vaccine is mostly humoral with little or no cellular immunity. Examples of inactivated vaccines: triple vaccine - DTP (diphtheria, tetanus, pertussis), hepatitis A, rabies, polio Salk type, meningococcal, typhoid, cholera. These vaccines require multiple doses to produce immunity and possibly require a booster to maintain its immunity.

\section{Subunit vaccines}

They are vaccines development for killed/inactivated agents. In this case, the particular vaccine contains an antigen (or antigens), it isn't composed of all antigens of immunogenic agent such as: hepatitis B, Haemophilus influenzae, influenza, pneumococcal, HPV.

\section{Live attenuated vaccines}

These vaccines are compound of live attenuated microorganisms in the laboratory; they must be able to multiply in the host organism so that it may occur the stimulation of an immune response. This immune response to the attenuated microorganism is identical for that one produced by natural infection because the immune system is unable to differentiate the infection by the vaccine microorganism or the wild microorganism. The multiplication of the vaccine microorganism is not usually capable of causing disease.
Examples of live attenuated vaccines: measles, mumps, rubella, polio Sabin vaccine, yellow fever, varicella, BCG, smallpox. These vaccines usually determine prolonged immunity with a single dose, with the exception of the polio vaccine.

\section{Adverse reaction to vaccines}

Local reactions are the most common symptoms and include pain, swelling and redness at the injection site. These reactions are usually mild and self-limited. However, on rare occasions can become severe (Arthus reactions).

Systemic reactions include fever, malaise, rash, myalgia, headache, and anorexia. These symptoms usually occur 1-2 weeks after the administration of live attenuated vaccines and they are considered to be a light "disease" caused by the multiplication of the vaccine microorganism. Allergic reactions are the most serious, including putting people's lives at risk but fortunately they are very rare.

\section{Contraindications to vaccination}

\section{General contraindications to vaccination:}

- Severe allergy to a previous dose of the vaccine

- Severe allergy to the components of the vaccine

- Moderate to severe acute illness

Note: The vaccine against measles, mumps, influenza (flu) and yellow fever are not recommended for individuals who are allergic to chicken egg protein.

\section{Contraindications to live attenuated vaccines:}

- Pregnancy, except in situations of high risk for the pregnant

- Presence of malignancy

- Congenital or acquired immunodeficiency

- Treatment with corticosteroids for immunosuppressive doses $(2 \mathrm{mg} / \mathrm{kg} /$ day in children and $20 \mathrm{mg} /$ day in adults for longer than 7 days), other types of immunosuppression (chemotherapy, radiotherapy)

- Recent blood product transfusion

\section{Contraindications to BCG vaccination:}

- All related to live attenuated vaccines

- Children weighing $<2 \mathrm{~kg}$ (technical impossibility of application)

\section{Contraindications for DPT vaccination}

(due to the pertussis component):

- Encephalopathy within 7 days post-vaccination

- Convulsion within 72 hours post-vaccination

- Persistent and inconsolable crying, with 3 hours or more in duration

\section{Indications for passive immunization}

The most common types of passive immunization are:

- Human Immunoglobulin for B Hepatitis 
- Human Immunoglobulin for rabies

- Human immunoglobulin for tetanus

- Human immunoglobulin for varicella zoster

- Human immunoglobulin combined: it's indicated for post exposure prophylaxis of hepatitis A and measles.

- Heterologous serum: diphtheria treatment, botulism treatment, tetanus and rabies prophylaxis.

\section{Vaccination dilemma versus immune disease}

In 2002, $\mathrm{Bach}^{3}$ in an interesting article published in the New England Journal of Medicine examined the fact that autoimmune diseases have increased significantly in correspondence to the decline of infectious diseases. Although infectious agents could trigger autoimmune diseases by antigenic mimicry, these are curious data obtained from epidemiological studies. Apparently infectious agents present superantigens that may have an action in the immune system, modulating it, and decreasing the expression of autoimmune diseases. How much vaccinations may influence this recent increase of autoimmune diseases is a matter to be clarified.

\section{Postvaccinal neurological complications}

There are several mechanisms that justify the complications. The immune mechanism is the most important, involving antigenic similarity with components of the nervous system or autoimmune diseases triggering in a predisposed individual. The example cited at the beginning of the chapter reinforces this impression. Nevertheless, it must not be forgotten that the agents in the attenuated vaccines can possibly be triggering the disease itself which we are trying to avoid. A typical example is the case of polio vaccination. In the 50s, when polio was an extremely common disease, vaccination by Sabin vaccine was highly useful mainly for two reasons: its effectiveness and the fact of having live attenuated virus life cycle very similar to wild virus, allowed the proliferation and excretion of the virus in feces of vaccinated individuals leading the vaccination of individuals from across the community. But today, when the risk for polio is very small, the Salk vaccine with inactivated virus is the most appropriate one because the risk of triggering polio is virtually zero. The oral polio vaccine should not be used in people with immunodeficiency (including asymptomatic HIV) or in people in contact with those individuals. Individuals with immunodeficiency, apart from the greater risk of polio vaccination, can eliminate the virus in feces for prolonged periods (months, years), which facilitates the occurrence of mutation ("reversal") and constitutes a risk to unvaccinated people. In a situation of low vaccine coverage in the population, the mutant virus can lead to epidemics or outbreaks of poliomyelitis, as occurred in Belarus (1965-66), Egypt (1983-93), the Dominican Republic and Haiti (2000-01), Philippines (2001), Madagascar (2001-02) and Nigeria (2005). The inactivated virus vaccine (Salk) should be used in Brazil from August 2013 onwards, to the beginning (first and second dose) of the immunization scheme suitable for children. Adults who have never been vaccinated, when traveling to high risk areas should receive the vaccine with inactivated virus (Salk), preferably at least the first two doses, for the risk of vaccinal poliomyelitis, which although it's small, is higher in this group than in children.

Other live attenuated virus vaccines, such as yellow fever should be used in an extremely restricted way in patients with potential immunological problems. The annual exacerbation rate following vaccination was 8.57 compared to 0.67 after the risk period ( 5 weeks after vaccination) in patients with multiple sclerosis ${ }^{4}$. Three months after immunization patients showed significant increase in lesional charge evidenced in T2-weighted sequences and gadolinium enhancing lesions. Therefore any vaccination for yellow fever in patients with multiple sclerosis, under immunomodulators or not, should be carefully evaluated.

Even newer vaccines with huge population impact have determined complications. Recent reports of neuromyelitis optica episodes and acute disseminated encephalomyelitis associated with HPV vaccination were published ${ }^{5-8}$.

Neurological complications after vaccination can reach any point of the entire nervous system from the brain up to muscles $^{9,10}$. In 1999, The American Academy of Neurology already described the risk of neurological complications in immunization procedures ${ }^{11}$.

\section{Brain}

a) Meningitis and encephalitis ${ }^{5,8,12-16}$ : Tenenbaum et al. ${ }^{16}$ in study of 84 children with an acute disseminated encephalomyelitis (ADEM) showed that $74 \%$ had a previous history of viral infection or vaccination. Classically ADEM is monophasic. However, in this survey 8 children (10\%) exhibited a biphasic behavior. The second relapse occurred with an average interval of 2.9 years. Evidently the basic differential diagnosis is with multiple sclerosis. Only the occurrence of an attack, often related to the infection, the absence of oligoclonal bands in cerebrospinal fluid at the first manifestation and relapse, in addition to non-appearance of new lesions in the extended follow-up suggest biphasic ADEM diagnostic.

In a survey during 20 years in the United States ${ }^{12}$ occurred 1396 cases of encephalitis after immunization. The responsible vaccines were: hepatitis B (354 cases, 25.5\%), influenza vaccination (208 cases, $14.9 \%$ ), vaccination for measles, mumps and rubella-MMR (208 cases, 14.9\%) and vaccination for Haemophilus influenza type B (120 cases - 8.6\%).

Another brain complication were ataxic forms ${ }^{15}$.

b) Demyelinating disease 4

c) Parkinsonism

d) Narcolepsy: Szakács et al. ${ }^{17}$ identified 37 children with narcolepsy. Nine of them started their symptoms before 
vaccination against $\mathrm{H} 1 \mathrm{~N} 1$ virus and 28 had onset of their symptoms related to vaccination. The average children age was 10 years. All affected after vaccination were positive for HLA DQB1*0602. These children also had very fast onset of their symptoms. This relationship had been related in some cases after streptococcal infection. Probably, the mimicry antigenic between the vaccine and cells producing hypocretin is the pathogenic mechanisms.

e) Encephalopathy

f) Epilepsy: there are descriptions of seizures and hypotonic/non-responsive episodes following vaccination for pertussis. Furthermore, there are several reports of seizures following influenza vaccination but the causality is not clear $^{18}$.

\section{Spinal cord}

a) Postvaccinal poliomyelitis: it was already discussed previously about the most recent use of the Salk vaccine, to the detriment of the Sabin vaccine.

b) Transverse myelitis: surveys of transverse myelitis reach to $28 \%$ of the cases as related to previous vaccinations ${ }^{19,20}$.

\section{Roots/nerves}

a) Poliradiculopathy: there are reports of polyradiculitis often associated with myelitis, after vaccination procedures, including toxoids ${ }^{21}$.

b) Guillain-Barré syndrome: it is related to a quite common complication described in vaccinations, particularly against influenza. There is even the possibility of recurrent episodes, a rare situation in usual clinical practice $^{14,22}$. c) Chronic inflammatory demyelinating polyradiculoneuropathy

d) Optic neuritis

e) Brachial and lumbosacral neuritis ${ }^{23}$

\section{Skeletal muscle}

a) Myositis: There are descriptions of complications probably caused by adjuvants, preservatives and contaminants (Macrophagic myofasciitis, for aluminum).

\section{Miscellany}

Neurotoxicity by adjuvant Thimerosal and new variant of Creutzfeldt-Jakob disease by material derived from infected cattle.

\section{Treatment}

The utilization of high-dose steroids (methylprednisolone $1 \mathrm{~g}$ IV for 3-5 days), with or without immunoglobulin (2 g/ kg/ $\mathrm{EV} /$ treatment) has shown satisfactory results in the treatment of immune diseases triggered by vaccinations ${ }^{24}$.

\section{Following the case report}

Due to relapse, repeated CSF in december/2011 which was normal, including oligoclonal bands absent. It was made methylprednisolone pulse therapy with improvement and discussed starting immunomodulator (44 ug betainterferonala thrice a week). She has showed severe hepatotoxicity and preferred to use no therapeutic regimen. On the last evaluation in march/2013, the patient was totally normal.

The doubt remains: multiphasic ADEM or multiple sclerosis?

\section{References}

1. Baxter D. Active and passive immunity, vaccine types,excipients and licensing. Occupational Medicine 2007;57:552-556.

2. Ministério da Saude - Fundação Nacional de Saúde. Manual de Normas de Vacinação 2001.

3. Bach J-F. The effect of infections on susceptibility to autoimmune allergic diseases. N Engl J Med 2002; 347 (12): 911-20.

4. Farez MF, Correale J. Yellow fever vaccination and increased relapse rate in travelers with multiple sclerosis. Arch Neurol. 2011 Oct; 68(10):1267-71.

5. Bomprezzi R. Acute disseminated encephalomyelitis following vaccination against human papilloma virus. Neurology 2010;74;864

6. Farrell FA. Comment: Human papillomavirus vaccination, induced autoimmunity, and neuromyelitis optica. Neurology 2012;79:287.

7. Menge T, Cree B, Saleh A., et al. Neuromyelitis optica following human papillomavirus vaccination. Neurology 2012;79:285.

8. Wildemann B, Jarius S, Hartmann M et al. Acute disseminated encephalomyelitis following vaccination against papilloma virus. Neurology 2009;72;2132-3.

9. MiravalleA, BillerJ,SchnitzlerE,Bonwit A. Neurological complications following vaccinations. Neurol Res. 2010 Apr; 32(3):285-92.
10. Piyasirisilp S, Hemachudha T. Neurological adverse events associated with vaccination. Curr Opin Neurol. 2002 Jun;15(3):333-8.

11. Fenichel GM. Assessment: Neurologic risk of immunization: Report of the Therapeutics and Technology Assessment Subcommittee of the American Academy of Neurology. Neurology 1999;52:1546-53.

12. Al Qudah Z, Abukwaik W, Patel H, Souayah N. Encephalitis after Vaccination in United States. A Report from the CDC/FDA Vaccine Adverse Event Reporting System. [1990-2010] (P03.151). Neurology April 22, 2012 78:P03.151.

13. Booss J, Davis LE. Smallpox and smallpox vaccination: Neurological implications. Neurology 2003;60;1241

14. Marchioni E, Ravaglia S, Piccolo G, Furione M, Zardini E, Franciotta D, Alfonsi E, Minoli L, Romani A, Todeschini A, Uggetti C, Tavazzi E, Ceroni M. Postinfectious inflammatory disorders: Subgroups based on prospective follow-up. Neurology 2005;65:1057-1065.

15. Sheikh Z, Abukwaik W, Souayah N. Ataxia after Vaccination in United States, a Report from the CDC/FDA Vaccine Adverse Event Reporting System [1990-2010] (P05.016). Neurology April 22, 2012 78:P05.016.

16. Tenembaum S, Chamoles N, Fejerman N. Acute disseminated encephalomyelitis A long-term follow-up study of 84 pediatric patients. Neurology 2002;59:1224-1231. 
17. Szakács A, Darin N, Hallböök T. Increased childhood incidence of narcolepsy in western Sweden after H1N1 influenza vaccination. Neurology 2013;80:1315-22.

18. Arnheim-Dahlström L, Hällgren J, Weibull CE, Sparén P. Risk of presentation to hospital with epileptic seizures after vaccination with monovalent AS03 adjuvanted pandemic A/H1N1 2009 influenza vaccine (Pandemrix): self controlled case series study. BMJ. 2012 Dec 18;345:7594.

19. Berman M, Feldman S, Alter M, et al. Acute transverse myelitis: Incidence and etiologic considerations. Neurology 1981;31;966-71.

20. Pidcock FS, Krishnan C, Crawford TO. Acute transverse myelitis in childhood: Center-based analysis of 47 cases. Neurology 2007; 68;1474-80.

21. Tezzon F, Tomelleri P, Ferrari G, Sergi A. Acute radiculomyelitis after antitetanus vaccination. Ital J Neurol Sci. 1994 May;15(4):191-3.

22. Seyal M, Ziegler DK, Couch JR. Recurrent Guillain-Barre syndrome following influenza vaccine. Neurology 1978; 28: 725-726.

23. Sander JE, Sharp FR. Lumbosacral plexus neuritis. Neurology 1981;31:470.

24. Fu DC, Montgomery JR. High-dose, rapid-infusion IVIG in postvaccination acute disseminated encephalomyelitis. Neurology $2008 ; 71 ; 294$ 